\title{
DVT following foot and ankle surgery: risk to the patient and surgeon
}

\author{
James D. F. Calder
}

Published online: 22 March 2013

(C) Springer-Verlag Berlin Heidelberg 2013

Debate continues as to how best protect our patients from suffering a venous thrombo-embolic event (VTE) following surgery. The risk following hip and knee arthroplasty is known to be significant, and there is consensus that steps should be taken to minimize this risk, but even here, there is conflict as to what methods should be used. The problem lies in deciding what type of VTE we trying to prevent and proving which method is most effective. Undoubtebly, below knee, deep vein thrombosis (DVT) carries a significant morbidity with regards to risk of post-thrombotic syndrome (including leg ulcers), but most orthopaedic studies concentrate on the prevention of pulmonary embolus (PE) and fatal complications rather than the longer term medical complications of DVT. Routine use of chemical prophylaxis with unfractionated heparin (UFH), low-molecular weight heparin (LMWH), factor Xa inhibitors and warfarin is widespread and has been shown to significantly reduce the risk of death from PE following hip and knee arthroplasty [13]. In addition to the economic cost from the routine use of pharmaceutical thrombo-prophylaxis, there are also significant potential side effects ranging from wound problems, increased infection rate and bleeding. Heparin-induced thrombocytopenia syndrome (HITS) is also a serious potential complication with an absolute risk in orthopaedic surgery of $0.2 \%$ for LMWH and $2.6 \%$ for UFH in a recent meta-analysis [6]. Mechanical methods may be effective in reducing VTE

J. D. F. Calder

The Chelsea and Westminster Hospital, 369 Fulham Road, London SW10 9NH, UK

J. D. F. Calder ( $\square)$

The Fortius Clinic, 17 Fitzhardinge St, London W1H 6EX, UK

e-mail: james.calder@fortiusclinic.com following hip and knee surgery, and they are also cost effective and safe [16].

How about foot and ankle surgery? Much of the advice has been extrapolated from data on hip and knee surgery, and as a result (in this increasingly litigious society), many surgeons routinely use chemical prophylaxis following ankle fractures, hindfoot surgery and Achilles tendon ruptures. Some even advocate its use following forefoot surgery. What is the evidence for this routine use, and what are the potential risks?

\section{Risk of VTE in foot and ankle surgery}

Shibuya et al. [12] reported low VTE rates specific to foot and ankle trauma from the National Data Trauma Bank. They concluded that the incidence of DVT and PE was 0.28 and $0.21 \%$, respectively. They noted that obesity was a significant risk factor with an odds ratio of 2.35 for DVT and 3.06 for PE. Age and increased injury severity scores had little effect on the incidence of VTE, and the authors concluded that routine use of pharmacological thromboprophylaxis is not necessary, but rather an individualized approach should be taken with assessment of risk factors. These patients were not screened for silent VTE, but Patil et al. [9] investigated 100 patients with ankle fractures treated in a cast. A colour duplex Doppler ultrasound examination was performed on removal of the cast. 5 DVTs were discovered (all symptomatic), one involved the femoral and another the popliteal vein. They concluded that routine use of chemical thrombo-prophylaxis was not indicated.

Jameson et al. [3] reported on complications following foot and ankle surgery from the large English NHS database. The respective incidence of DVT and PE was 0.12 
and $0.17 \%$ following ankle fracture, 0.01 and $0.02 \%$ following first metatarsal osteotomy and 0.03 and $0.11 \%$ following hindfoot fusion. The incidence of PE following 1,633 total ankle replacements was $0.06 \%$ with no DVTs being reported. There would have inevitably been underreporting as none of these patients were routinely screened for DVT, and asymptomatic VTE would have been missed. However, the overall risk of significant DVT would indeed appear low following foot and ankle surgery. This viewpoint is supported by our recent review of 2,654 patients where the incidence of VTE following all forms of foot and ankle surgery was $0.42 \%$ (27 patients were lost to followup, and if these are included, the worst case scenario for VTE was $1.43 \%$ ) [1].

Treatment of Achilles tendon injuries, however, stands out as a major risk factor for VTE. Lapidus et al. [5] reported the occurrence of DVT in more than $1 / 3$ of patients following surgical treatment of Achilles tendon rupture in 105 consecutive patients screened with duplex Doppler. Nilsson-Helander [7] reported a similar rate in a prospective ultrasound study of 100 patients after Achilles rupture. Some of the patients in the Lapidus [11] study were included in a follow-up report 7 years after documentation of their DVT and an incidence of mild post-thrombotic syndrome was found in $11 \%$ and DVT recurrence in $2 \%$. Healy reported a symptomatic VTE rate following Achilles rupture as similar to that following hip arthroplasty with DVT and PE rates of 4.8 and $1.4 \%$, respectively [2]. Countering this is a recent retrospective data base analysis of 1,172 patients with Achilles tendon rupture where the overall rates for DVT and PE were reported as 0.43 and $0.34 \%$, respectively [8]. Obesity, age and surgery did not affect the incidence of VTE, and the authors concluded that routine chemical thrombo-prophylaxis is not indicated.

\section{Which chemical agents are the most effective in foot and ankle surgery?}

Lapidus et al. [4] performed a randomized, double-blind, placebo-controlled study following ankle fractures investigating the protective effect of Dalteparin in 272 patients. Phlebography was performed on removal of the cast. Although the incidence of VTE was high $(21 \%$ Dalteparin and $28 \%$ placebo), there was no significant difference between the groups, and the majority of the DVTs were distal and asymptomatic. They were unable to demonstrate any protective effect when using Dalteparin following ankle fractures. Despite the fact that DVT is common following surgery for Achilles tendon rupture, in their randomized, placebo-controlled study, Lapidus et al. [5] also failed to demonstrate any significant reduction in the incidence of DVT when using Dalteparin. There are also theoretical risks of using factor Xa inhibitors following Achilles rupture as thrombin is a key component in the healing tendon, and neutralized thrombin has been shown to half the strength of tendon repair in animal models [14], whereas intermittent use of low-molecular weight heparin does not appear to effect tendon healing [15]. Griffiths et al. [1] failed to demonstrate a reduction in rate of VTE with aspirin following foot and ankle surgery, and Pelet et al. [10] reported that thrombo-prophylaxis did not affect the incidence of VTE unless there were specific risk factors.

In summary, the incidence of symptomatic VTE following foot and ankle surgery is generally low (approximately $0.5 \%$ ), but the risk of complications when using chemical prophylaxis is not insignificant (HITS may effect 0.2-2.6\% with LMWH and UFH) [6]. With this low rate of VTE, many of the current studies are underpowered and are unable to identify which groups are particularly at risk and, more importantly, which method of chemoprophylaxis is the most effective. Therefore, routine use of such agents is questionable. Earlier mobilization regimens and the routine use of mechanical measures may suffice and reduce the risk of VTE to an acceptable level. However, an individualized approach should be taken as those who are obese, have a previous history of DVT or have an Achilles tendon rupture probably are in a high risk category and may require specific chemoprophylaxis. I am sure that the debate will continue as the true risk-benefit of chemical prophylaxis is probably blurred a little by pressure from the pharmaceutical industry and lawyers!

\section{References}

1. Griffiths JT, Matthews L, Pearce CJ, Calder JD (2011) Incidence of venous thromboembolism in elective foot and ankle surgery with and without aspirin prophylaxis. J Bone Joint Surg Br 94(2): 210-214

2. Healy B, Beasley R, Weatherall M (2010) Venous thromboembolism following prolonged cast immobilisation for injury to the tendo Achillis. J Bone Joint Surg Br 92(5):646-650

3. Jameson SS, Augustine A, James P, Serrano-Pedraza I, Oliver K, Townsend D, Reed MR (2011) Venous thromboembolic events following foot and ankle surgery in the English National Health Service. J Bone Joint Surg Br 93(4):490-497

4. Lapidus LJ, Ponzer S, Elvin A, Levander C, Larfars G, Rosters S, de Bri E (2007) Prolonged thromboprophylaxis with Dalteparin during immobilization after ankle fracture surgery: a randomized placebo-controlled, double-blind study. Acta Orthop 78(4): $528-535$

5. Lapidus LJ, Rosters S, Ponzer S, Levander C, Elvin A, Larfars G, de Bri E (2007) Prolonged thromboprophylaxis with dalteparin after surgical treatment of Achilles tendon rupture: a randomized, placebo-controlled study. J Orthop Trauma 21(1):52-57

6. Martel N, Lee J, Wells PS (2005) Risk for heparin-induced thrombocytopenia with unfractionated and low-molecular-weight heparin thromboprophylaxis: a meta-analysis. Blood 106(8):2710 
7. Nilsson-Helander K, Thurin A, Karlsson J, Eriksson BI (2009) High incidence of deep venous thrombosis after Achilles tendon rupture: a prospective study. Knee Surg Sports Traumatol Arthrosc 17(10):1234-1238

8. Patel A, Ogawa B, Charlton T, Thordarson D (2012) Incidence of deep vein thrombosis and pulmonary embolism after Achilles tendon rupture. Clin Orthop Relat Res 470(1):270-274

9. Patil S, Gandhi J, Cursor I, Hi AC (2007) Incidence of deep-vein thrombosis in patients with fractures of the ankle treated in a plaster cast. J Bone Joint Surg Br 89(10):1340-1343

10. Pelet S, Roger ME, Belzile EL, Bouchard M (2012) The incidence of thromboembolic events in surgically treated ankle fracture. J Bone Joint Surg Am 94-A:502-506

11. Persson LM, Lapidus LJ, Larfars G, Rosters S (2011) Deep venous thrombosis after surgery for Achilles tendon rupture: a provoked transient event with minor long-term sequelae. J Thromb Haemost 9(8):1493-1499

12. Shibuya N, Frost $\mathrm{CH}$, Campbell JD, Davies ML, Jupiter DC (2012) Incidence of acute deep vein thrombosis and pulmonary embolism in foot and ankle trauma: analysis of the National Trauma Data Bank. J Foot Ankle Surg 51(1):63-68

13. Sobieraj DM, Coleman CI, Tongbram V, Chen W, Colby J, Lee S, Kluger J, Making S, Ashaye A, White CM (2011) Comparative effectiveness of low-molecular-weight heparins versus other anticoagulants in major orthopedic surgery: a systematic review and meta-analysis. Pharmacotherapy 32(9):799-808

14. Virchenko $\mathrm{O}$, Grenegård $\mathrm{M}$, Aspenberg $\mathrm{P}$ (2006) Independent and additive stimulation of tendon repair by thrombin and platelets. Acta Orthop 77(6):960-966

15. Virchenko O, Aspenberg P, Lindahl TL (2008) Low molecular weight heparin impairs tendon repair. J Bone Joint Surg $\mathrm{Br}$ 90(3):388-392

16. Zhao JM, He ML, Xiao ZM, Li TS, Wu H, Jiang H (2012) Different types of intermittent pneumatic compression devices for preventing venous thromboembolism in patients after total hip replacement. Cochrane Database Syst Rev 14(11):CD009543 